\title{
ZUM MECHANISMUS MASSENSPEKTROMETRISCHER FRAGMENTIERUNGSREAKTIONEN.-I METHOXYGRUPPENWANDERUNGEN IN DEN MASSENSPEKTREN DER METHYLÄTHER VON LINEAREN UND VERZWEIGTEN ALIPHATISCHEN POLYALKOHOLEN.
}

\author{
HANS-Friedrich GrützMacher und JohanN WinkLeR \\ Chemisches Staatsinstitut-Institut für Organische Chemie der Universität \\ Hamburg, 2 Hamburg-13, Papendamm 6, Deutschland
}

(Received 4 March 1968; accepted 12 March 1968) \begin{abstract}
are observed in the mass spectra of compounds of the following type:

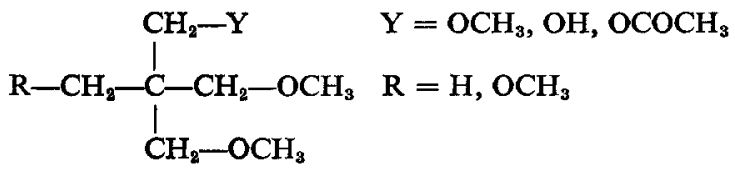

Abstract-The formation of 1,1-dimethoxy-alkyl rearrangement ions in the mass spectra of methyl ethers of linear diols, 1,2,3-triols and of derivatives of pentaglycerol and pentaerythritol has been investigated by deuterium labelling and mass measurements. Methoxy group migrations do not occur, or at least only to a small amount, in the mass spectra of the diol-dimethyl ethers. The mass spectra of methyl ethers of 1,2,3-triols exhibit characteristic peaks of the rearrangement ions $+\mathrm{CH}\left(\mathrm{OCH}_{3}\right)_{2}$ and $+\mathrm{CR}\left(\mathrm{OCH}_{3}\right)_{2}$. These ions arise by a 1,3-migration of a methoxy group, probably during a one step degradation of the molecular ion to give a molecule methyl alkenyl ether and a $\mathrm{H}$-atom or alkyl radical as neutral fragments. Large peaks of the rearrangement ion $+\mathrm{CH}\left(\mathrm{OCH}_{3}\right)_{2}$

A radical ion, formed by loss of $\mathrm{HY}$ from the molecular ion, rearranges by methoxy group migration in the mass spectra of these substances. Rearrangement by migration of hydroxy and acetoxy groups are also observed, but no migration of a Cl-atom.

Zusammenfassung-Die Bildung von 1,1-Dimethoxyalkyl-Umlagerungsionen in den Massenspektren der Methyläther von linearen Diolen, 1,2,3-Triolen und Derivaten der verzweigten Polyole Pentaglycerin und Pentaerythrit wird mit Hilfe von Markierungen mit Deuterium und Massefeinbestimmungen untersucht. Methoxygruppenwanderungen treten in den Massenspektren der Dioldimethyläther nicht oder nur in sehr geringem Ausmaß auf. Die Massenspektren der 1,2,3-Trioltrimethyläther besitzen aber charakteristische Peaks der Umlagerungsionen ${ }^{+} \mathrm{CH}\left(\mathrm{OCH}_{3}\right)_{2}$ und ${ }_{+} \mathrm{CR}\left(\mathrm{OCH}_{3}\right)_{2}$. Diese Ionen entstehen durch eine 1,3-Wanderung einer Methoxygruppe, wahrscheinlich bei einem einstufigen Zerfall der Molekül-Ionen unter Bildung von Methyl-alkenyl-äther und H-Atom bzw. Alkylradikal als neutrale Fragmente. Große Peaks der Umlagerungsionen $+\mathrm{CH}\left(\mathrm{OCH}_{3}\right)_{2}$ werden in den Massenspektren von Verbindungen des Typs<smiles>[R]CC(C[Y])(COC)COC</smiles>

gefunden. Bei diesen Verbindungen wandert eine Methoxygruppe beim weiteren Zerfall von Radika]Ionen, die durch Abspaltung von HY aus den Molekül-Ionen entstehen. Wanderungen von Hydroxy- und Acetoxy-Gruppen werden ebenfalls beobachtet, aber keine Wanderung von ClAtomen. 
WANDERUNGEN von Atomen und Atomgruppen während des durch ElektronenstoB induzierten Zerfalls organischer Moleküle besitzen besonderes Interesse. Zeigen diese Umlagerungsreaktionen doch, daß organische Ionen in der Gasphase nicht nur durch eine Folge einfacher Bindungsspaltungen und Eliminierungen kleiner stabiler Fragmente zerfallen, sondern ebenso wie in Lösungen auch kompliziertere Reaktionen eingehen können. Da ferner in den Umlagerungsionen Atome miteinander verknüpft sind, die im Molekül nicht durch chemische Bindungen aneinander gekettet sind, kann ihre Bildung massenspektrometrische Strukturbestimmungen erschweren. Dies gilt vor allem für Umlagerungsreaktionen, bei denen die wandernde Gruppe eine funktionelle Gruppe ist und Heteroatome enthält. Im allgemeinen sind Fragment-Ionen, welche die Heteroatome des Moleküls enthalten, strukturspezifische Schlüsselfragmente des Massenspektrums.

In der letzten Zeit wurden mehrfach massenspektrometrische Umlagerungsreaktionen beobachtet, ${ }^{1-7}$ bei denen eine Hydroxy-Gruppe oder eine der davon abgeleiteten Gruppen $\mathrm{CH}_{3} \mathrm{O}-, \mathrm{CH}_{3} \mathrm{COO}$ - und $\left(\mathrm{CH}_{3}\right)_{3} \mathrm{SiO}-$ wandert. Am längsten ist die Wanderung von $\mathrm{CH}_{3} \mathrm{O}$-Gruppen bekannt, die von Heyns und Müller ${ }^{1}$ in den Massenspektren permethylierter Methyl-pentopyranoside nachgewiesen wurde. Die Massenspektren dieser Verbindungen zeigen einen großen Peak bei der $\mathrm{MZ}(=$ Massenzahl) 75. Gleiches gilt auch für die Massenspektren anderer O-methylierter Zuckerderivate, die einen Halbacetalring besitzen. ${ }^{8}$ Die Ionen der MZ 75 sind Dimethoxymethyl-Ionen $a$, die eigentlich für die Massenspektren von Dimethylacetalen typisch sind:

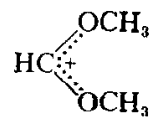

a $\mathrm{MZ} 75$

In den Massenspektren der permethylierten Methyl-pyranoside entstehen die Ionen $a$ bevorzugt aus der glykosidischen $\mathrm{CH}-\mathrm{OCH}_{3}$-Gruppe und einem $\mathrm{CH}_{3} \mathrm{O}$-Substituenten, der aus der Position 3 des Pyranosidringes wandert. Als treibende Kraft für diese Umlagerungsreaktion wurde die Bildung des Ions $a$ angesehen, in dem die positive Ladung durch Mesomerie gut stabilisiert ist. ${ }^{9}$ Ferner wurde angenommen, daß eine Methoxygruppenwanderung an eine cyclische Acetalstruktur im Molekül gebunden ist. In den Massenspektren der Methyläther von Inositen und linearen Zuckeralkoholen ${ }^{10}$ werden jedoch ebenfalls große Peaks der Umlagerungsionen $a$ beobachtet. Folglich sind weder eine Acetalgruppe noch ein Ring im Molekül Vorbedingung für das Eintreten der Methoxygruppenwanderung. Daher wurden jetzt die Massenspektren von Methyläthern linearer, verzweigter und cyclischer Polyole untersucht mit dem Ziel, das in diesen Verbindungen für die Methoxygruppenwanderung verantwortliche Strukturelement aufzufinden. In dieser Arbeit wird über die Massenspektren der Methyläther offenkettiger Polyole berichtet, bei denen eine Fragmentierung durch Methoxygruppenwanderungen besonders überraschend ist.

1. MASSENSPEKTREN VON METHYLÄTHERN LINEARER POLYOLE

Die einfachsten Verbindungen, in deren Massenspektren DimethoxymethylUmlagerungsionen des Typs $a$ auftreten könnten, sind die Methyläther linearer Diole. In Tabelle 1 sind die größeren Peaks der Massenspektren der Methyläther 
von Diolen des Typs $\mathrm{HOCH}_{2}-\left(\mathrm{CH}_{2}\right)_{n}-\mathrm{CH}_{2} \mathrm{OH}$ mit $n=1$ bis 4 (I bis IV), von Butan- (V), Hexan- (VI) und 2-Äthylhexan-1,3-diol (VII) aufgeführt. Die Massenspektren dieser Verbindungen bieten keine Besonderheiten.

Wie für die Massenspektren aliphatischer Polyäther zu erwarten war, ist die Intensität der Molekül-Ionen sehr gering. Die größeren Peaks können Ionen zugeordnet werden, die durch $\alpha$-Spaltungen und/oder Eliminierungen von Methanol entstehen:

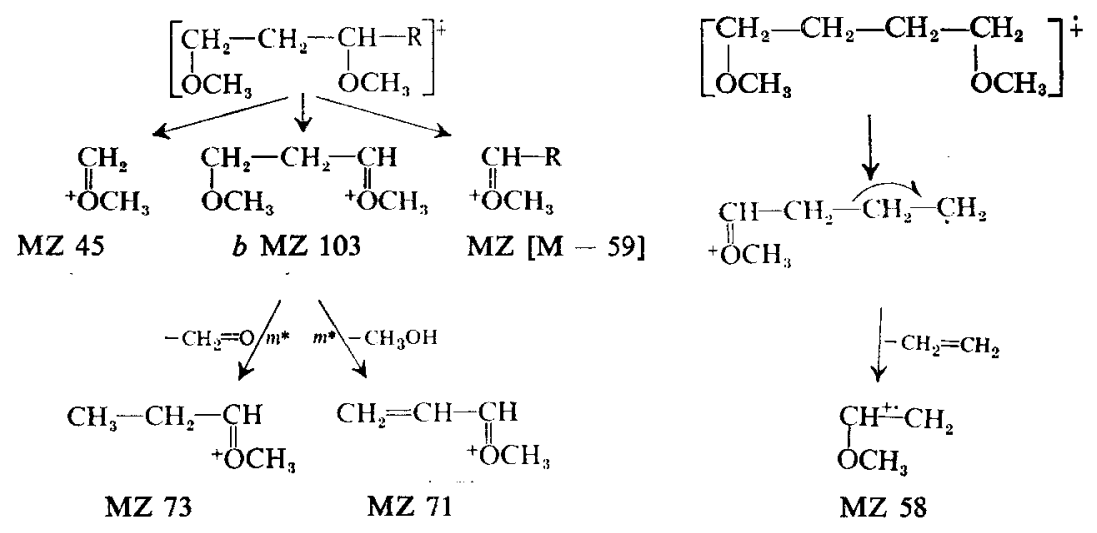

Kleine, aber deutliche Peaks der MZ 75 lassen sich nur in den Massenspektren der Derivate der 1,3-Diole I, V, VI und VII nachweisen. Massebestimmungen (Tabelle 2) zeigen, daß sie durch $\mathrm{C}_{3} \mathrm{H}_{7} \mathrm{O}_{2}{ }^{+}$-Ionen entstehen, die durch Methoxygruppenwanderungen gebildet werden müssen. Ein einfacher Bildungsmechanismus wäre eine 1,3-Wanderung einer Methoxygruppe in $b$ unter Eliminierung von Äthylen:

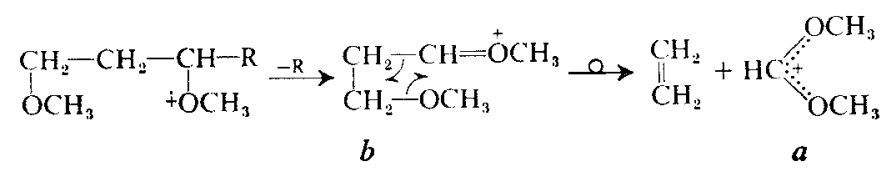

Nach diesem Bildungsmechanismus ist zu erwarten, daß die Intensität der Umlagerungsionen a steigt, wenn die Bildung der Ionen $b$ durch Abspaltung eines größeren Alkylradikals aus den Molekül-Ionen begünstigt wird, also in der Reihe I, V, VII und VI. Dies trifft nicht $z u$. Die Intensität der Ionen $a$ ist aber so gering und ihre Bildung für die Massenspektren der 1,3-Dioldimethyläther so wenig charakteristisch, $\mathrm{da}$ auf eine eingehende Untersuchung verzichtet wurde. Immerhin zeigt aber die geringe Intensität der Ionen $a$ in den Massenspektren der Methyläther der 1,3-Diole trotz der Möglichkeit zur Bildung eines stabilen Äthylenfragments bei der Methoxygruppenwanderung, daß der Energiegewinn bei der Bildung des Umlagerungsions $a$ alleine für diese Reaktion nicht ausreicht.

Besonders deutlich wird dies im Massenspektrum des Butan-1,2,4-triol-trimethyläthers (VIII, Abb. 1), das wegen der energetisch günstigen Abspaltung eines $\mathrm{CH}_{3} \mathrm{O}-$ $\mathrm{CH}_{2}$-Radikals einen großen Peak des Ions b bei der MZ 103 besitzt. Trotzdem ist auch hier der Peak der Umlagerungsionen $a$ klein. Die Ionen $b$ zerfallen bevorzugt durch Eliminierung von Methanol zu Ionen der MZ 71 (metastabiler Peak gef. 49,0 , berechn. 48,94) und durch Abspaltung von Formaldehyd zu Ionen der MZ 73 (metastabiler Peak gef. 51,8, berechn. 51,74). 
Das Fehlen eines größeren Peaks der MZ 75 im Massenspektrum von VIII ist von Interesse, weil in der Reihe der linearen Zuckeralkohole der Methyläther des Erythrits die Verbindung mit der kleinsten C-Zahl ist, deren Massenspektrum einen

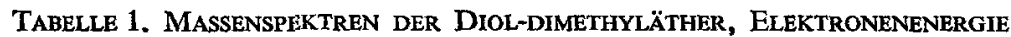
$70 \mathrm{eV}$, Ionenquellentemperatur $200^{\circ} \mathrm{C}$, Probentemperatur $150-175^{\circ} \mathrm{C}$,

\begin{tabular}{|c|c|c|c|c|c|c|c|}
\hline $\mathbf{M Z}$ & $\stackrel{I}{M}=104$ & $\begin{array}{c}\text { II } \\
M=118\end{array}$ & $\stackrel{\text { III }}{M=132}$ & $\begin{array}{c}I V \\
M=146\end{array}$ & $\begin{array}{c}V \\
M=118\end{array}$ & $\begin{array}{c}V I \\
M=146\end{array}$ & $\begin{array}{c}\text { VII } \\
M=174\end{array}$ \\
\hline 39 & 4 & 5 & 3,5 & 2,5 & 8 & 5,5 & 7,5 \\
\hline 40 & - & - & - & - & - & - & - \\
\hline 41 & 12,5 & 10 & 11 & 12 & 18 & 15,5 & 21,5 \\
\hline 42 & 16 & 5 & 5 & 5 & 5,5 & 6 & 2,5 \\
\hline 43 & 4,5 & 6,5 & 3 & 3,5 & 17 & 21 & 6,5 \\
\hline 44 & - & - & - & - & - & - & - \\
\hline 45 & $\underline{100}$ & $\underline{100}$ & $\underline{100}$ & $\underline{100}$ & 85 & $\underline{100}$ & $\underline{100}$ \\
\hline 54 & & & & 14,5 & 2 & & \\
\hline 55 & & 9 & 6 & 8,5 & 11,5 & 21 & 18 \\
\hline 56 & & 6 & 2 & 5 & 5,5 & 6,5 & 3,5 \\
\hline 57 & 13 & 2 & 2,5 & 2 & 3 & 10 & 5,5 \\
\hline 58 & 2,5 & 88 & 18 & 12 & 7,5 & 6 & 2 \\
\hline 59 & 2 & 4 & 2 & 2 & $\underline{100}$ & 8 & 2,5 \\
\hline 67 & & & 8,5 & 16 & & 4 & 3 \\
\hline 68 & & & 23,5 & 3 & & & \\
\hline 69 & & & 6 & 4 & & 3 & 4,5 \\
\hline 71 & 19 & 10 & 65 & 38 & 35,5 & 36 & 12,5 \\
\hline 72 & 67 & 2 & 5,5 & & 2 & 7,5 & \\
\hline 73 & 4,5 & & & & 4 & 3 & \\
\hline 75 & 3,5 & 0,5 & 0,5 & 0,5 & 2,5 & 1,0 & 1,5 \\
\hline 82 & & & & 29,5 & & 8 & \\
\hline 83 & & & & 5 & & 6 & \\
\hline 85 & & 3,5 & 5 & 6 & 3 & 6,5 & 5,5 \\
\hline 86 & & 10 & & & 40 & 3 & 12 \\
\hline 87 & & & 3 & 2 & 4 & 18 & 47,5 \\
\hline 88 & & 2 & & & 3 & 2 & 3 \\
\hline 100 & & & 32 & & & 2,5 & \\
\hline 103 & & 8 & & & 4,5 & 18 & \\
\hline 113 & & & & & & & 3 \\
\hline 114 & & & & 9 & & 9 & \\
\hline 131 & & & & & & 2 & 6,5 \\
\hline 142 & & & & & & & 3,5 \\
\hline
\end{tabular}

großen Peak für die Umlagerungsionen $a$ enthält. In Analogie zu den Massenspektren der Methyläther verweigter Polyole (s.u.) könnte nämlich auch bei den linearen Polyolen eine Methoxygruppenwanderung in einem Radikal-Ion vermutet werden, das durch 1,4-Eliminierung von Methanol aus dem Molekül-Ion entsteht:<smiles></smiles> 
Tabelle 2. Massefeinbestimmungen, Bezugssubstanz Perfluorokerosene (Columbia Organic Chemicals, Co., Inc.)

\begin{tabular}{|c|c|c|c|}
\hline Verbindung & gef. Masse & berechn. Masse & Summenformel \\
\hline I & 75,0449 & 75,0446 & $\mathrm{C}_{3} \mathrm{H}_{7} \mathrm{O}_{2}$ \\
\hline $\mathbf{v}$ & 75,0441 & 75,0446 & $\mathrm{C}_{3} \mathrm{H}_{7} \mathrm{O}_{2}$ \\
\hline IX & 75,0450 & 75,0446 & $\mathrm{C}_{3} \mathrm{H}_{7} \mathrm{O}_{2}$ \\
\hline $\mathbf{x}$ & $\begin{array}{r}71,0496 \\
72,0574 \\
73,0651 \\
75,0450 \\
85,0652 \\
103,0758\end{array}$ & $\begin{array}{r}71,0496 \\
72,0575 \\
73,0653 \\
75,0446 \\
85,0653 \\
103,0759\end{array}$ & $\begin{array}{l}\mathrm{C}_{4} \mathrm{H}_{7} \mathrm{O} \\
\mathrm{C}_{4} \mathrm{H}_{8} \mathrm{O} \\
\mathrm{C}_{4} \mathrm{H}_{9} \mathrm{O} \\
\mathrm{C}_{3} \mathrm{H}_{7} \mathrm{O}_{2} \\
\mathrm{C}_{5} \mathrm{H}_{8} \mathrm{O} \\
\mathrm{C}_{5} \mathrm{H}_{11} \mathrm{O}_{2}\end{array}$ \\
\hline $\mathrm{XI}$ & $\begin{array}{r}75,0445 \\
86,0735 \\
117,0911\end{array}$ & $\begin{array}{r}75,0446 \\
86,0732 \\
117,0915\end{array}$ & $\begin{array}{l}\mathrm{C}_{3} \mathrm{H}_{7} \mathrm{O}_{2} \\
\mathrm{C}_{6} \mathrm{H}_{10} \mathrm{O} \\
\mathrm{C}_{6} \mathrm{H}_{13} \mathrm{O}_{2}\end{array}$ \\
\hline XII & $\begin{array}{l}59,0497 \\
71,0502 \\
73,0297(50 \%) \\
73,0660(50 \%) \\
75,0442\end{array}$ & $\begin{array}{l}59,0497 \\
71,0496 \\
73,0290 \\
73,0653 \\
75,0446\end{array}$ & $\begin{array}{l}\mathrm{C}_{3} \mathrm{H}_{7} \mathrm{O} \\
\mathrm{C}_{4} \mathrm{H}_{7} \mathrm{O} \\
\mathrm{C}_{3} \mathrm{H}_{5} \mathrm{O}_{2} \\
\mathrm{C}_{4} \mathrm{H}_{8} \mathrm{O} \\
\mathrm{C}_{3} \mathrm{H}_{7} \mathrm{O}_{2}\end{array}$ \\
\hline XIII & $\begin{array}{c}75,0442 \\
85,0652 \\
103,0546(65 \%) \\
103,0759(35 \%) \\
133,0865 \\
135,0805 \\
165,0914\end{array}$ & $\begin{array}{r}75,0446 \\
85,0653 \\
103,0548 \\
103,0759 \\
133,0865 \\
135,0810 \\
165,0915\end{array}$ & $\begin{array}{l}\mathrm{C}_{3} \mathrm{H}_{7} \mathrm{O}_{2} \\
\mathrm{C}_{5} \mathrm{H}_{9} \mathrm{O} \\
\mathrm{C}_{8} \mathrm{H}_{7} \\
\mathrm{C}_{5} \mathrm{H}_{11} \mathrm{O}_{2} \\
\mathrm{C}_{8} \mathrm{H}_{13} \mathrm{O}_{3} \\
\mathrm{C}_{9} \mathrm{H}_{11} \mathrm{O} \\
\mathrm{C}_{10} \mathrm{H}_{13} \mathrm{O}_{2}\end{array}$ \\
\hline$X V$ & 75,0438 & 75,0446 & $\mathrm{C}_{3} \mathrm{H}_{7} \mathrm{O}_{2}$ \\
\hline XVI & $\begin{array}{r}75,0442 \\
101,0598 \\
85,0653\end{array}$ & $\begin{array}{r}75,0446 \\
101,0603 \\
85,0653\end{array}$ & $\begin{array}{l}\mathrm{C}_{3} \mathrm{H}_{7} \mathrm{O}_{2} \\
\mathrm{C}_{5} \mathrm{H}_{8} \mathrm{O}_{2} \\
\mathrm{C}_{5} \mathrm{H}_{8} \mathrm{O}\end{array}$ \\
\hline XVII & $\begin{array}{l}58,0418 \\
71,0498 \\
75,0435\end{array}$ & $\begin{array}{l}58,0419 \\
71,0496 \\
75,0446\end{array}$ & $\begin{array}{l}\mathrm{C}_{3} \mathrm{H}_{6} \mathrm{O} \\
\mathrm{C}_{4} \mathrm{H}_{7} \mathrm{O} \\
\mathrm{C}_{3} \mathrm{H}_{7} \mathrm{O}_{2}\end{array}$ \\
\hline XVIII & $\begin{array}{l}55,0549(90 \%) \\
57,0339(90 \%) \\
61,0287 \\
75,0444\end{array}$ & $\begin{array}{l}55,0548 \\
57,0340 \\
61,0290 \\
75,0446\end{array}$ & $\begin{array}{l}\mathrm{C}_{4} \mathrm{H}_{7} \\
\mathrm{C}_{3} \mathrm{H}_{5} \mathrm{O} \\
\mathrm{C}_{2} \mathrm{H}_{5} \mathrm{O}_{2} \\
\mathrm{C}_{3} \mathrm{H}_{7} \mathrm{O}_{2}\end{array}$ \\
\hline XIX & $\begin{array}{r}72,0574 \\
75,0445 \\
86,0728 \\
103,0394\end{array}$ & $\begin{array}{r}72.0575 \\
75,0446 \\
86,0732 \\
103,0395\end{array}$ & $\begin{array}{l}\mathrm{C}_{4} \mathrm{H}_{8} \mathrm{O} \\
\mathrm{C}_{3} \mathrm{H}_{7} \mathrm{O}_{2} \\
\mathrm{C}_{5} \mathrm{H}_{10} \mathrm{O} \\
\mathrm{C}_{4} \mathrm{H}_{7} \mathrm{O}_{3}\end{array}$ \\
\hline
\end{tabular}


Die gegenüber den 1,3-Diol-dimethyläthern nur unwesentlich größere Intensität der Ionen a im Massenspektrum von VIII spricht aber gegen diese Zerfallsreaktion.

Deutlicher als in den Massenspektren der Methyläther von 1,3-Diolen und von VIII tritt ein Peak der MZ 75 im Massenspektrum des Glycerin-trimethyläthers (IX)

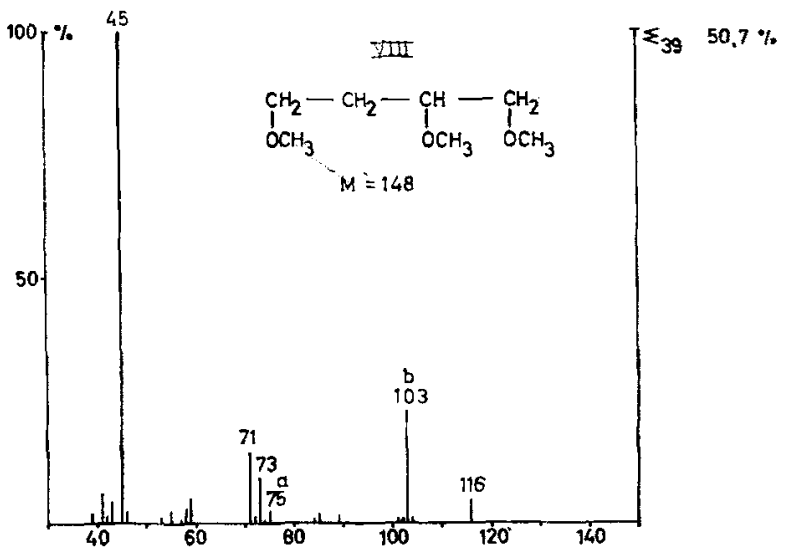

ABB. 1. Massenspektrum des Butan-1.2.4-triol-trimethyläthers, $70 \mathrm{eV}$.

hervor (Abb. 2). Die Zuordnung dieses Peaks zu den Ionen $a$ ergibt sich aus der Elementzusammensetzung $\mathrm{C}_{3} \mathrm{H}_{7} \mathrm{O}_{2}$ (Tabelle 2) und der vollständigen Verschiebung des Peaks zur MZ 81 im Massenspektrum des Tris-(trideuteromethyl)-äthers des Glycerins (IXa). Die gleiche Verschiebung im Massenspektrum des Glycerin-1,3-diO-trideuteromethyl-2-O-methyläthers (IXb) zeigt, daB die Ionen a durch eine 1,3Wanderung einer Methoxygruppe entstehen.

Ebenso wie bei den 1,3-Diol-dimethyläthern wird auch bei IX die Intensität der Ionen $a$ durch Substitution eines der H-Atome am C-1 durch eine Alkylgruppe nur wenig erhöht. Sie steigt von $7,4 \%\left(1,5 \% \Sigma_{39}\right)$ bei IX auf $11,2 \%\left(2,9 \% \Sigma_{39}\right)$ im Massenspektrum des Pentan-1,2,3-triol-trimethyläthers (X, Abb. 3) und 8,8\% $\left(2,6 \% \Sigma_{39}\right)$ in dem des Hexan-1,2,3-triol-trimethyläthers (XI, Abb. 4). Massebestimmungen (Tabelle 2) und die Verschiebung des Peaks zur MZ 81 im Massenspektrum des Tris-(trideuteromethyl)-äthers (Xa) von $X$ bestätigen auch bei diesen Verbindungen die Struktur der Ionen $a$.

Im Massenspektrum von X fällt ein Peak der MZ 103 auf, der entsprechend der größeren Alkylgruppe von XI im Massenspektrum dieser Verbindung bei der MZ 117 gefunden wird. Massebestimmungen (Tatelle 2) ergeben die Zusammensetzung $\mathrm{C}_{5} \mathrm{H}_{11} \mathrm{O}_{2}$ bzw. $\mathrm{C}_{6} \mathrm{H}_{13} \mathrm{O}_{2}$, diese Ionen besitzen daher zwei Methoxygruppen. Dies wird bestätigt durch eine vollständige Verschiebung zur MZ 109 im Massenspektrum des Trideuteromethyläthers Xa. Dagegen beobachtet man im Massenspektrum des Trimethyläthers von Pentan-1,2,3-triol-d $\mathrm{d}_{1}-(3)(\mathrm{Xb})$ keine Verschiebung des Peaks der MZ 103, so daß das $\mathrm{H}$-Atom am C-3 nicht mehr in diesen Ionen vorhanden ist. Danach müssen die Ionen der MZ 103 bzw. 117 die Strukturen von 1,1-Dimethoxypropyl- bzw. 1,1-Dimethoxybutyl-Fragmenten besitzen und Homologe des Umlagerungsions $a$ sein. Sie entstehen wie dieses durch Methoxygruppenwanderung, für 




(a)

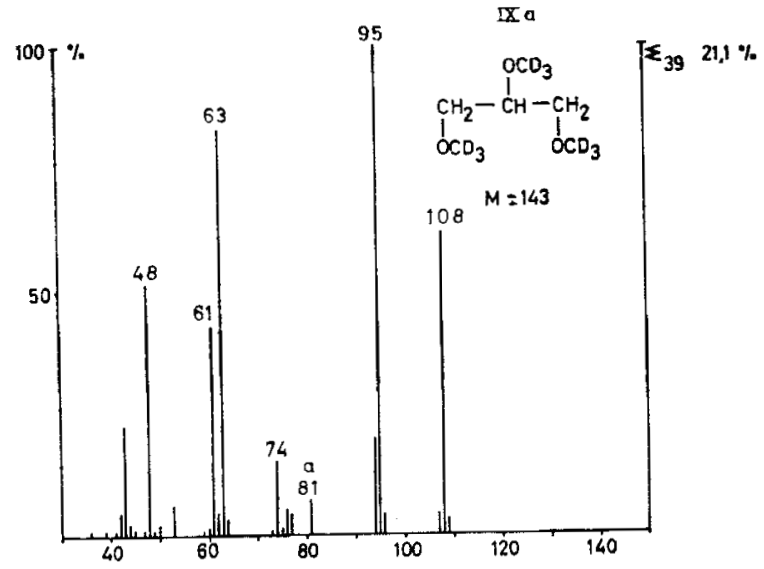

(b)

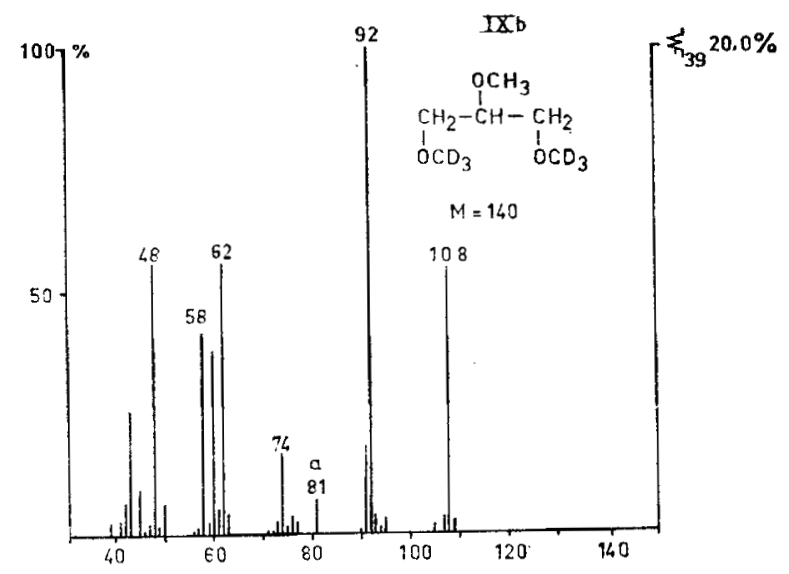

(c)

ABв. 2. (a) Massenspektrum des Glycerin-trimethyläthers, $70 \mathrm{eV}$. (b) Massenspektrum des Glycerin-tris-(trideuteromethyl)-äthers, $70 \mathrm{eV}$. (c) Massenspektrum des Glycerin-1,3-di-O-trideuteromethyl-2-O-methyläthers, $70 \mathrm{eV}$. 




(a)

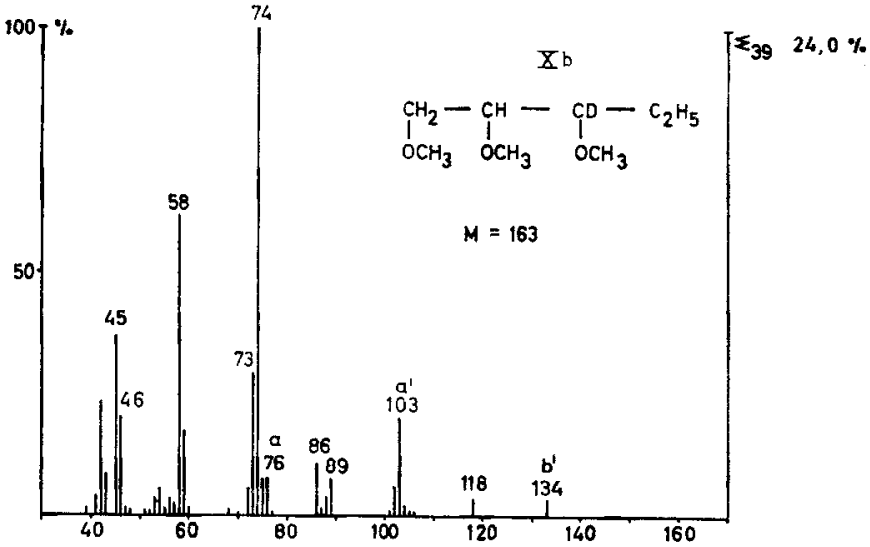

(b)

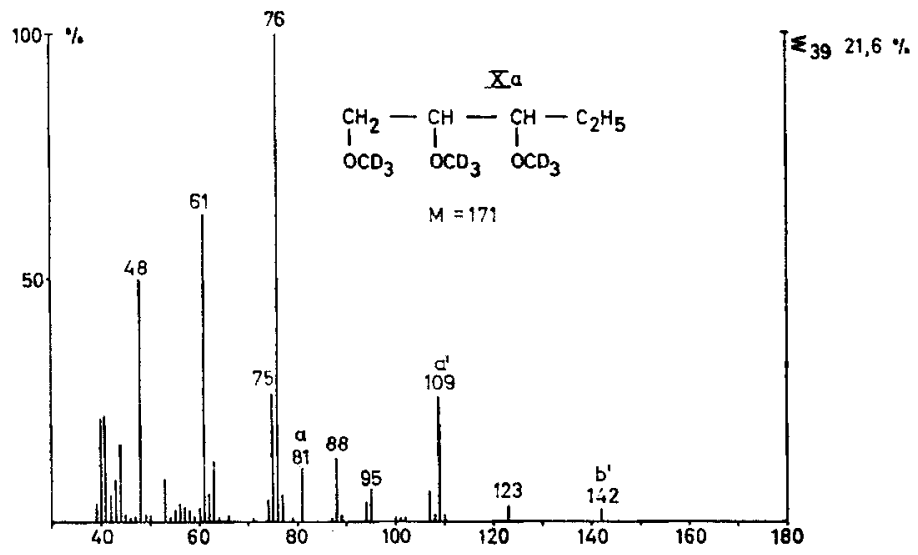

(c)

ABB. 3. (a) Massenspektrum des Pentan-1,2,3-triol-trimethyläthers, $70 \mathrm{eV}$. (b) Massenspektrum des Pentan-1,2,3-triol-d $\mathrm{d}_{1}$-(3)-trimethyläthers, $70 \mathrm{eV}$. (c) Massenspektrum des Pentan-1,2,3-triol-tris-(trideuteromethyl)-äthers, $70 \mathrm{eV}$. 


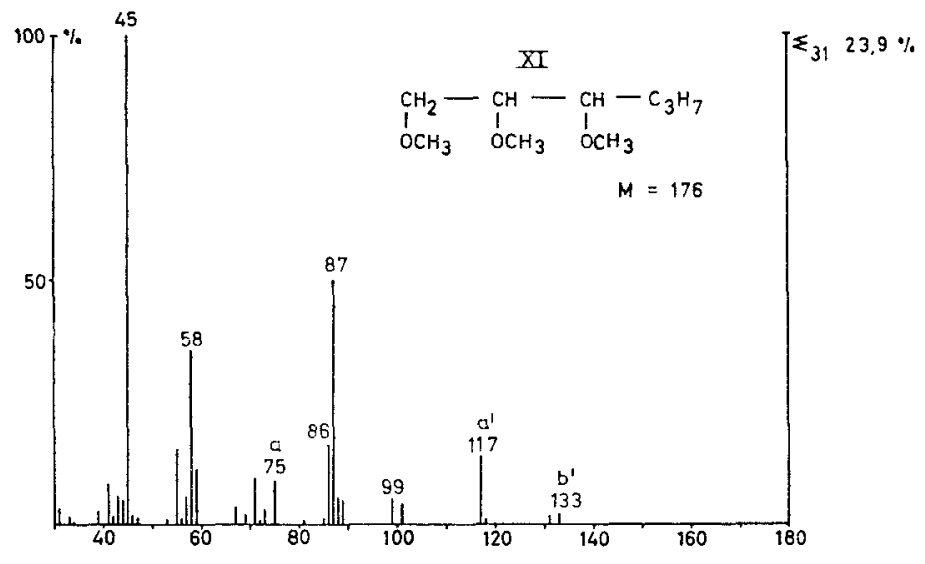

Aвв. 4. Massenspektrum des Hexan-1,2,3-triol-trimethyläthers, $70 \mathrm{eV}$.

die folgender zweistufiger Mechanismus angenommen werden kann:

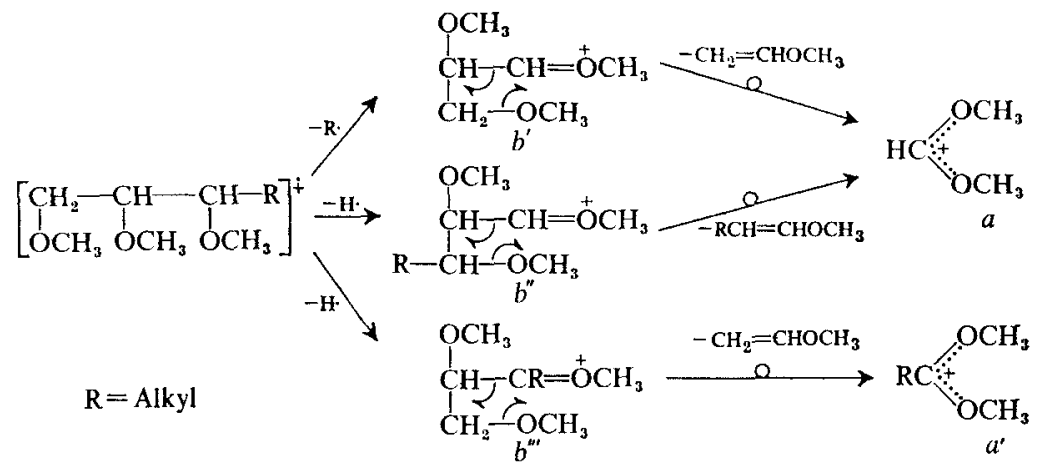

Im ersten Reaktionsschritt wird aus dem Molekül-Ion entweder die Alkylgruppe zum Ion $b^{\prime}$ oder ein H-Atom zu den Ionen $b^{\prime \prime}$ oder $b^{\prime \prime \prime}$ abgespalten. Es schließt sich eine 1.3-Wanderung der Methoxygruppe zum positiv geladenen Zentrum an, die bei $b^{\prime}$ und $b^{\prime \prime}$ zu den Umlagerungsionen a führt, bei $b^{\prime \prime \prime}$ die Umlagerungsionen $a^{\prime}$ ergibt. Dieser Reaktionstyp, Wanderung einer Methoxygruppe zum positiven Zentrum in einem geradelektronischen Fragment-Ion, ist bereits in den Massenspektren der Methylester von $\beta$-Brom- $\beta$-phenyl-propionsäure und $\alpha$-Brom- $\alpha$-phenylessigsäure beobachtet worden. ${ }^{4}$

Ưberraschend und mit einem zweistufigen Bildungsmechanismus nicht ohne weiteres vereinbar ist die große Intensität der Ionen $a^{\prime}$, die mit $27,4 \%\left(7,0 \% \Sigma_{39}\right)$ bei $\mathrm{X}$ und $14,0 \%\left(4,0 \% \Sigma_{39}\right)$ bei XI die der Ionen a übertrifft. Da die Abspaltung einer Alkylgruppe aus dem Molekül-Ion energetisch günstiger ist als die Abspaltung eines $\mathrm{H}$-Atoms, beobachtet man in den Massenspektren von X und XI deutliche Peaks der Ionen $b^{\prime}$, während die Intensität der Ionen $b^{\prime \prime}$ und $b^{\prime \prime \prime}$ sehr gering ist. Entsprechend sollte eigentlich die Intensität der aus $b^{\prime}$ entstehenden Ionen $a$ die der Ionen $a^{\prime}$ übertreffen. 
Die positive Ladung wird in dem Ion $a^{\prime}$ durch den induktiven Effekt der Alkylgruppe noch besser stabilisiert als im Ion $a$. Dieser Effekt könnte zu einer bevorzugten Bildung der Ionen $a^{\prime}$ führen, wenn Methoxygruppenwanderung im Molekül-Ion vor einer Fragmentierung eintritt, z.B. durch einen Platzwechsel von Methoxygruppe und H-Atom in 1,3-Stellung zu den Zwischenstufen $c$ und $c^{\prime}$. Die Spaltung der C-2/C-3Bildung in $c^{\prime}$ ist dann günstiger als die Spaltung der C-1/C-2-Bindung in $c$ :

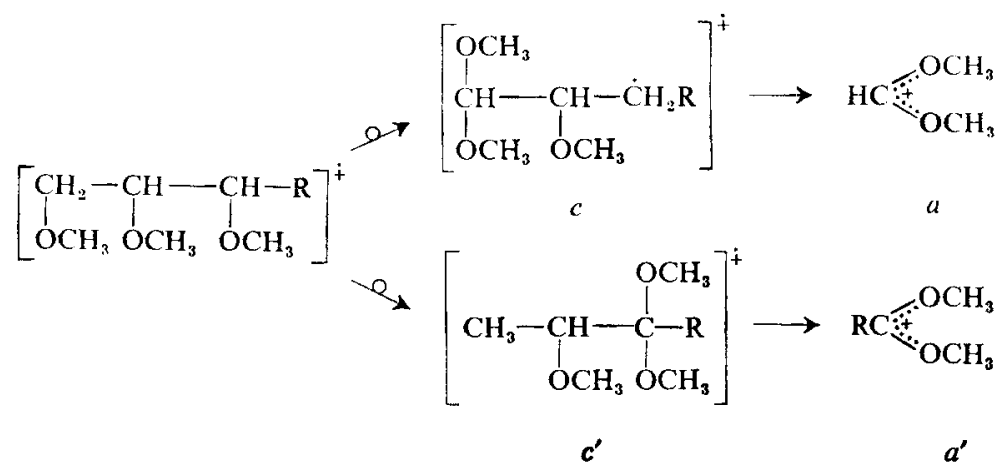

Nach diesem Mechanismus müßten die Ionen $a$ ausschließlich über die Zwischenstufe $c$ entstehen und das C-1 des Triol-Moleküls enthalten. Das Massenspektrum von $\mathrm{Xb}$ zeigt aber, daß $72 \%$ der Ionen a aus dem C-3 des Triols entstehen, so daß dieser Reaktionsmechanismus ausgeschlossen werden kann.

Man muß daher annehmen, daß die Bildung der Umlagerungsionen $a$ und $a^{\prime}$ nicht durch einen zweistufigen Zerfall erfolgt, sondern bevorzugt in einer einstufigen Reaktion, bei der Wanderung und Abspaltung entweder des H-Atoms oder der Alkylgruppe vom C-3 synchron verlaufen. In diesem Fall kann der für die Bildung eines $\mathrm{H}$-Atoms notwendige Mehraufwand an Energie durch die größere Stabilität des Ions $a^{\prime}$ kompensiert werden. Die dritte Methoxygruppe am C-2 erleichtert die Spaltung der C-2/C-3-Bindung in diesem einen Reaktionsschritt. Da diese Gruppe in den Methyläthern der 1,3-Diole fehlt, wird auch die Bildung der Umlagerungsionen $a$ und $a^{\prime}$ unterdrückt:

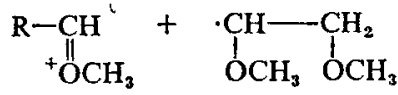

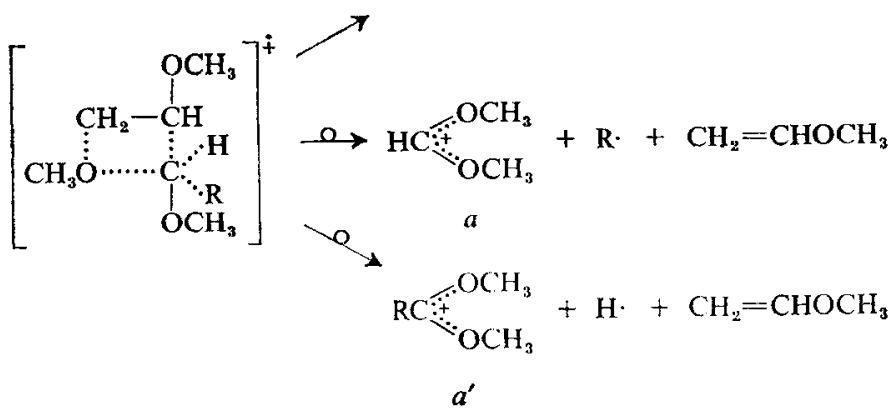

Im Gegensatz zu dem auf Seite 303 diskutierten zweistufigen Reaktionsmechanismus erfolgt die Methoxygruppenwanderung nicht mehr in einem geradelektronischen 
Fragment-Ion, sondern in einem Molekül-Ion, bei dem der Sitz der positiven Ladung und des Radikalelektrons nicht genau angegeben werden kann. Wahrscheinlich wird aber die Umlagerung durch einen 'Elektronenmangel' am C-3 ausgelöst. Dadurch wird die für die Wanderung notwendige Konformation des angeregten Molekül-Ions begünstigt. In welche Richung der Zerfall aus diesem Anregungszustand heraus erfolgt, wird dann durch die Stabilität der entstehenden Bruchstücke bestimmt.

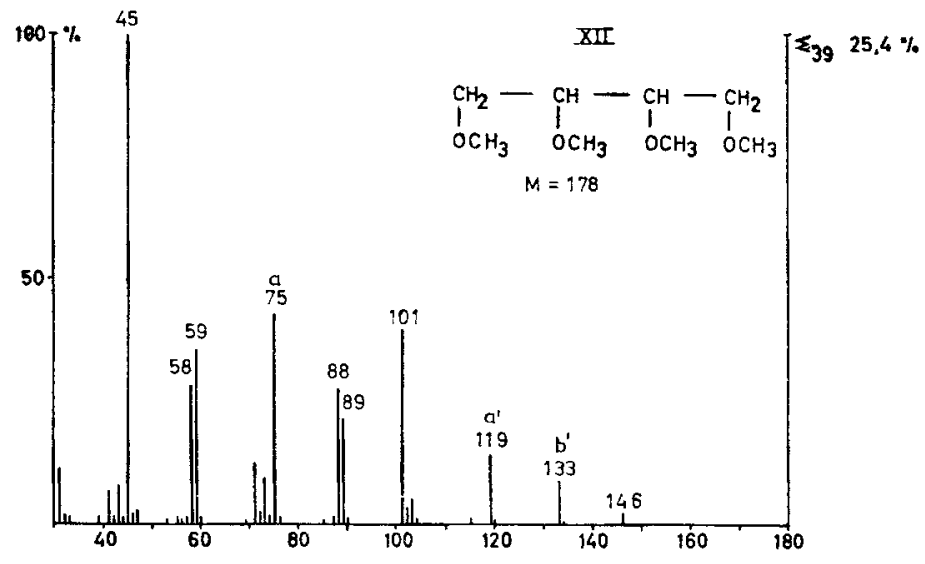

AвB. 5. Massenspektrum des Erythrit-tetramethyläthers, $70 \mathrm{eV}$.

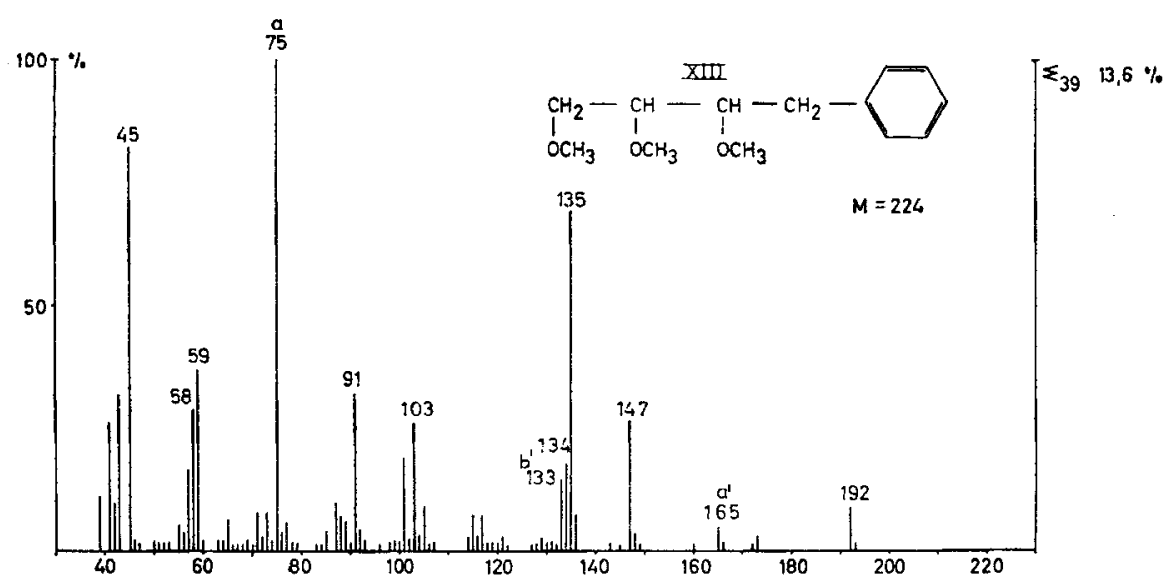

AB8. 6. Massenspektrum des 4-Phenyl-butan-1.2.3-triol-trimethyläthers, $70 \mathrm{eV}$.

Mit diesen Vorstellungen im Einklang sind die Massenspektren der Methyläther des Erythrits (XII, Abb. 5) und des 4-Phenyl-butan-1,2,3-triols (XIII, Abb. 6). Sie besitzen Peaks der Umlagerungsionen $a$ bei der MZ 75 und der Umlagerungsionen $a^{\prime}$ bei den MZ 119 bzw. 165. Die Zuordnung der Peaks wird durch die Verschiebungen der Peaks um 6 bzw. 9 Masseneinheiten im Massenspektrum des Tetra-(trideuteromethyl)-äthers XIIa und durch die Elementzusammensetzung der Ionen (Tabelle 2) bestätigt. Die Reste $\mathrm{R}$ können bei XII und XIII als stabile $\mathrm{CH}_{3} \mathrm{OCH}_{2}$ - und 
$\mathrm{C}_{6} \mathrm{H}_{5} \mathrm{CH}_{2}-$ Radikale abgespalten werden. Die Energiebilanz für die Bildung der Ionen $a$ aus den angeregten Molekül-Ionen wird dann so günstig, daß nun der Energiegewinn durch die Stabilisierung der positiven Ladung in den Ionen $a^{\prime}$ nicht mehr zur Kompensation der energetisch ungünstigen Abspaltung eines H-Atoms ausreicht. Die Intensität der Ionen $a$ steigt daher bei (XII) auf $42,7 \%\left(10,8 \% \Sigma_{39}\right)$, im Spektrum von (XIII) bilden sie den Hauptpeak $\left(13,6 \% \Sigma_{39}\right)$.

Bei der Bildung der Umlagerungsionen $a$ aus den Molekül-Ionen der Methyläthern von Pentiten und Hexiten werden ebenso wie bei XII stabile MethoxyalkylRadikale abgespalten, so daß die Massenspektren dieser Verbindungen ebenfalls

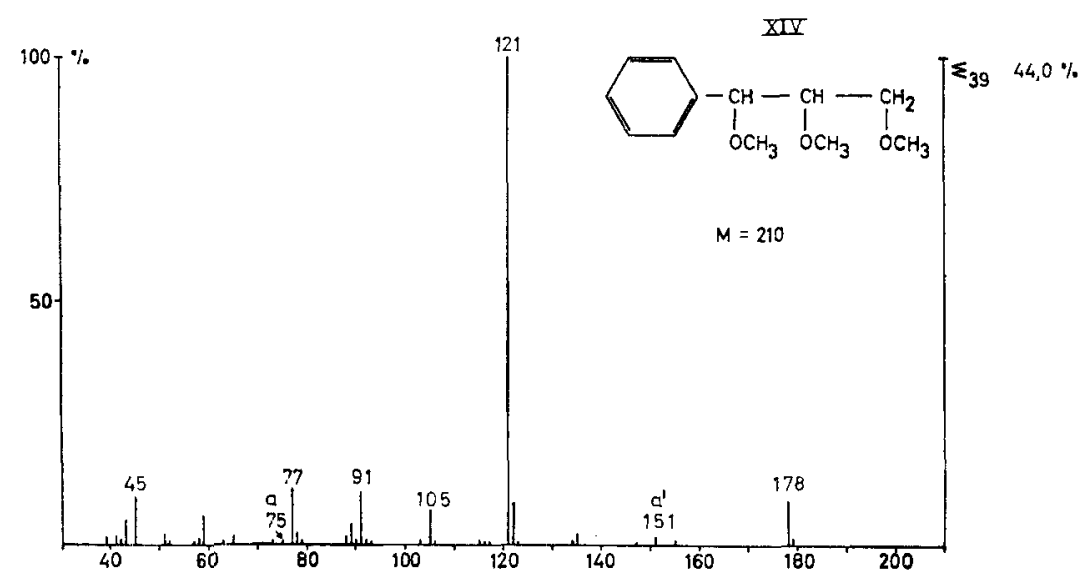

Aвв. 7. Massenspektrum des 3-Phenyl-propan-1.2.3-triol-trimethyläthers, $70 \mathrm{eV}$.

große Peaks der MZ 75 besitzen. Daneben beobachtet man auch kleine Peaks der Ionen $a^{\prime}$ bei den MZ 163 und 207.9

Eine bevorzugte Bildung der Ionen $a^{\prime}$ könnte für das Massenspektrum des Methyläthers von 3-Phenyl-propan-1,2,3-triol (XIV) erwartet werden, weil mit $\mathrm{R}=$ Phenyl die positive Ladung in diesem Ion besonders gut stabilisiert wird. Wie das Massenspektrum (Abb. 7) aber zeigt, wird bei XIV die C-2/C-3-Bindung unter Bildung eines $\alpha$-Methoxybenzyl-Ions der $m / e 121$ so leicht gespalten, daß diese Reaktion allen anderen bevorzugt wird. Die Peaks der Umlagerungsionen $a$ und $a^{\prime}$ bei den MZ 75 und MZ 151 sind daher klein.

Ebenso wie in den Massenspektren der Methyläther der Diole beobachtet man auch in den Massenspektren der Verbindungen IX bis XIV die für Polymethoxyverbindungen erwarteten Fragmentierungsreaktionen. So entstehen charakteristische Peaks durch Ionen der MZ 45, MZ 89, MZ 133, MZ [M - 45] und MZ [M - 89], die durch $\alpha$-Spaltungen gebildet werden:

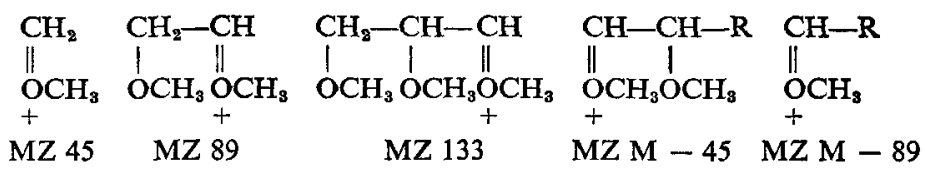

Aus diesen Ionen wird in einem zweiten Reaktionsschritt Formaldehyd (bevorzugt bei den Ionen der MZ 89) oder Methanol (bevorzugt bei den Ionen der MZ 133) 
eliminiert. Besonders groß ist die Intensität der Ionen der MZ 45. Im Massenspektrum der am C-3 deuterierten Verbindung Xb wird ein Drittel dieses Peaks zur MZ 46 verschoben. Die Ionen der MZ 45 entstehen daher nich tnur durch Spaltung der C-1/C-2-Bindung, sondern auch aus den Ionen $\mathrm{MZ}[\mathrm{M}-89]$ durch Eliminierung eines Olefinmoleküls:

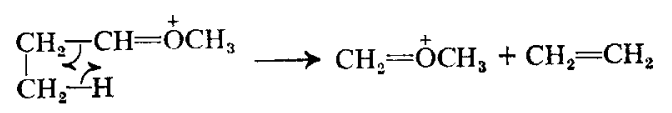

Ein größerer Peak für die Ionen $\left[\mathrm{M}-\mathrm{CH}_{3} \mathrm{OH}\right]$ wird nur bei den Verbindungen XIII und XIV beobachtet, bei denen eine Stabilisierung der Doppelbindung durch Konjugation mit dem Benzolring möglich ist. Eine thermische Methanolabspaltung läßt sich jedoch nicht ganz ausschließen. Methanolabspaltung der primären Methoxygruppe durch eine 1,4-Eliminierung und Spaltung der C-2/C-3-Bindung erklärt die Bildung von Ionen mit der Struktur von Methyl-alkenyl-äthern:

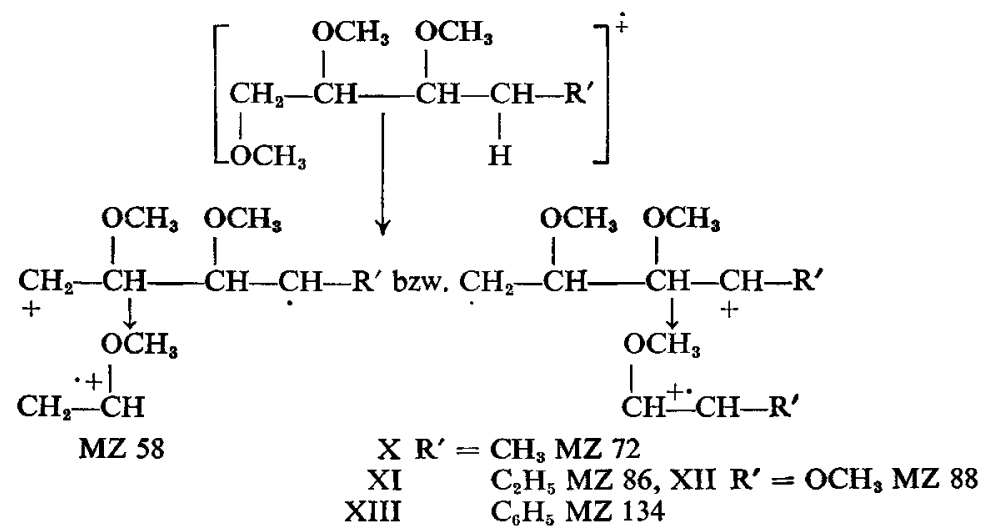

2. MASSENSPEKTREN VON METHYLÄTHERN VERZWEIGTER POLYOLE

Vergleicht man die Massenspektren der Methyläther von 2,2-Dimethylpropan-1,3diol (XV, Abb. 8), Pentaglycerin (XVI, Abb. 9) und Pentaerythrit (XVII, Abb. 10),

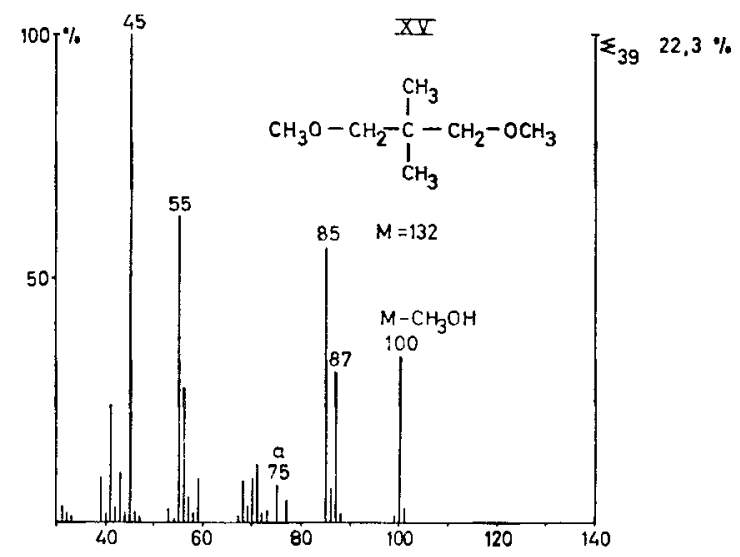

ABB. 8. Massenspektrum des 2.2-Dimethyl-propan-1.3-diol-dimethyläthers, $70 \mathrm{eV}$ 
so erkennt man deutlich den großen Einfluß, den die dritte Methoxygruppe in einer Seitenkette in 1,3-Stellung zu den anderen Methoxygruppen auf die Fragmentierungsreaktionen besitzt. Das Massenspektrum von XV zeigt ebenso wie die der übrigen 1,3-Diol-dimethyläther nur einen kleinen Peak der Umlagerungsionen $a$, und die Fragment-Ionen mit großer Intensität entstehen durch einfache Bindungsspaltungen. Die Anwesenheit der dritten Methoxygruppe in XVI bewirkt bereits

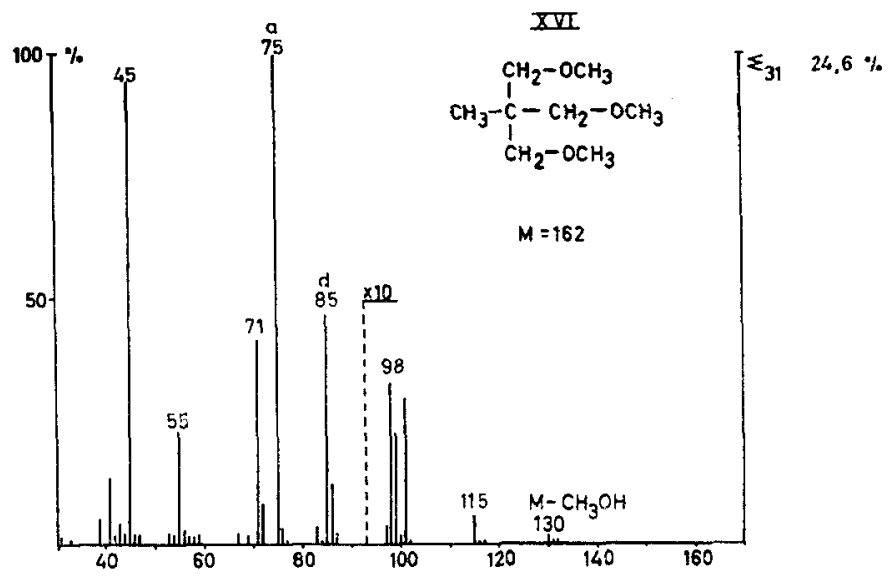

ABв. 9. Massenspektrum des Pentaglycerin-trimethyläthers, $70 \mathrm{eV}$.

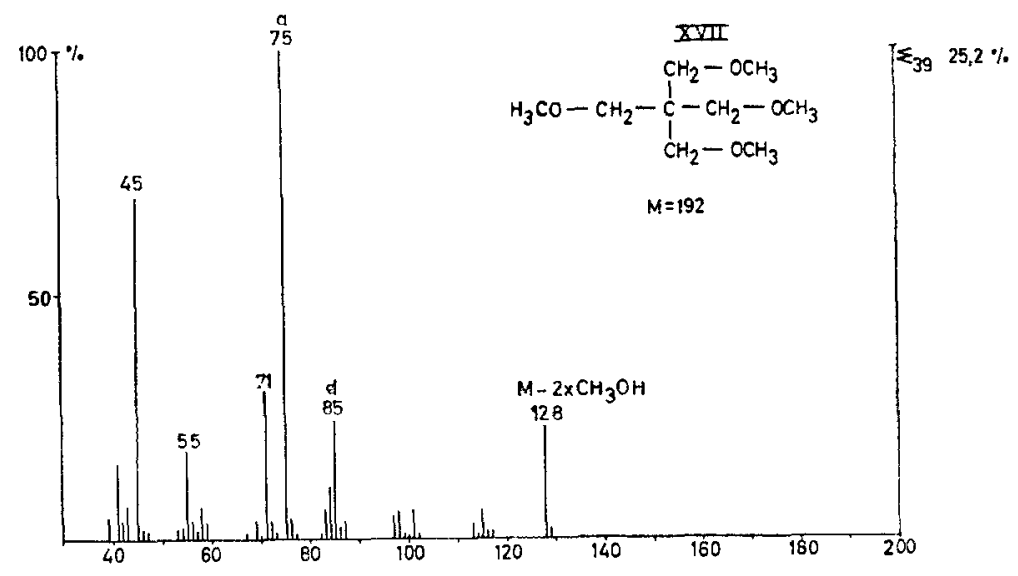

AвB. 10. Massenspektrum des Pentaerythrit-tetramethyläthers, $70 \mathrm{eV}$.

einen Intensitätsanstieg der Ionen $a$ um das Zehnfache, so daß der Peak der MZ 75 zum Hauptpeak wird. Im Massenspektrum von XVII, das 4 Methoxygruppen in 1,3-Stellung zueinander besitzt, beherrscht der Peak der MZ 75 das Spektrum.

Im Vergleich zu XV sind in XVI und XVII 1,3-Wanderungen der Methoxygruppen statistisch begünstigt. Dieser Faktor ist jedoch nicht die Ursache für die große Intensität der Ionen $a$. In den Massenspektren des Pentaglycerin-dimethyläthers (XVIII) und des Pentaglycerin-dimethyläther-monoacetats (XIX) sind ebenfalls 
große Peaks der MZ 75 vorhanden, obwohl diese Verbindungen wie XV nur zwei Methoxygruppen besitzen (Abb. 11 und 12). Wie bei XVII ist der Peak der MZ 75 auch im Massenspektrum des Pentaerythrit-trimethyläthers (XX, Abb. 13) noch sehr groß.

Gemeinsam ist den Verbindungen XVI bis XX, daß sie außer zwei Methoxygruppen in 1,3-Stellung zueinander mindestens einen weiteren Substituenten $Y$

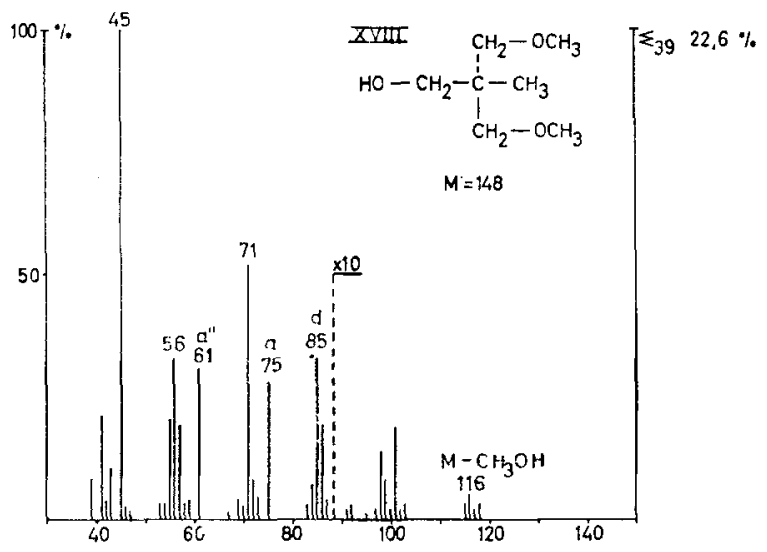

ABB. 11. Massenspektrum des Pentaglycerin-dimethyläthers, $70 \mathrm{eV}$.

$\left(\mathrm{OH}, \mathrm{OCH}_{3}\right.$ oder $\left.\mathrm{OCOCH}_{3}\right)$ besitzen, der aus dem Molekül-Ion als HY eliminiert werden kann. XV zeigt in seinem Massenspektrum bei der MZ 100 einen deutlichen Peak für die Ionen $\left[\mathrm{M}-\mathrm{CH}_{3} \mathrm{OH}\right.$ ]. Bei den übrigen Verbindungen ist dagegen der Peak der Ionen [M - HY] sehr klein. Diese Ionen zerfallen schnell weiter, entweder durch eine Methoxygruppenwanderung oder durch Abspaltung eines $\mathrm{CH}_{3} \mathrm{OCH}_{2}-\mathrm{Radikals}$ :
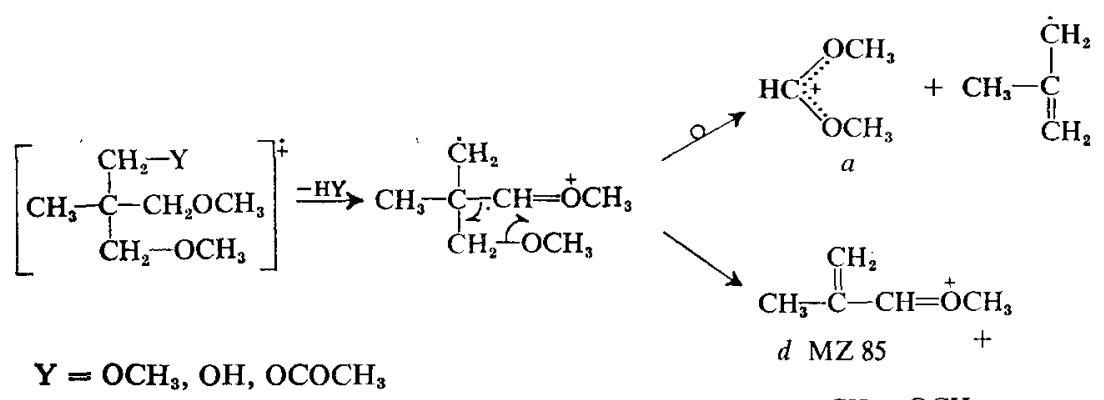

$$
\cdot \mathrm{CH}_{2}-\mathrm{OCH}_{3}
$$

Während in den Massenspektren der Methyläther linearer 1,2,3-Triole die Umlagerungsionen a in einem Reaktionsschritt aus den angeregten Molekül-Ionen gebildet werden, erfolgt die Methoxygruppenwanderung bei XVI bis XX nach einer Methanolabspaltung in einem Radikal-Ion, in dem die positive Ladung durch CarbeniumOxenium-Ion-Mesomerie stabilisiert ist. Das Radikalelektron ist aber am primären C-Atom in einer energetisch ungünstigen Position. Die treibende Kraft für die Umlagerungsreaktion ist daher nicht nur eine zusätzliche Stabilisierung der positiven 
Ladung im Umlagerungsion $a$, sondern vor allem die Stabilisierung des Radikalelektrons im mesomeriefähigen Butenylradikal. Diese Umlagerung ist daher das Gegenstück zur Methoxygruppenwanderung in den Massenspektren $\operatorname{der} \beta$-Brom- $\beta$ phenyl-propionsäure- und $\alpha$-Brom- $\alpha$-phenyl-essigsäuremethylester, ${ }^{4}$ die mit Sicherheit in einem geradelektronischen Fragment-Ion eintritt.

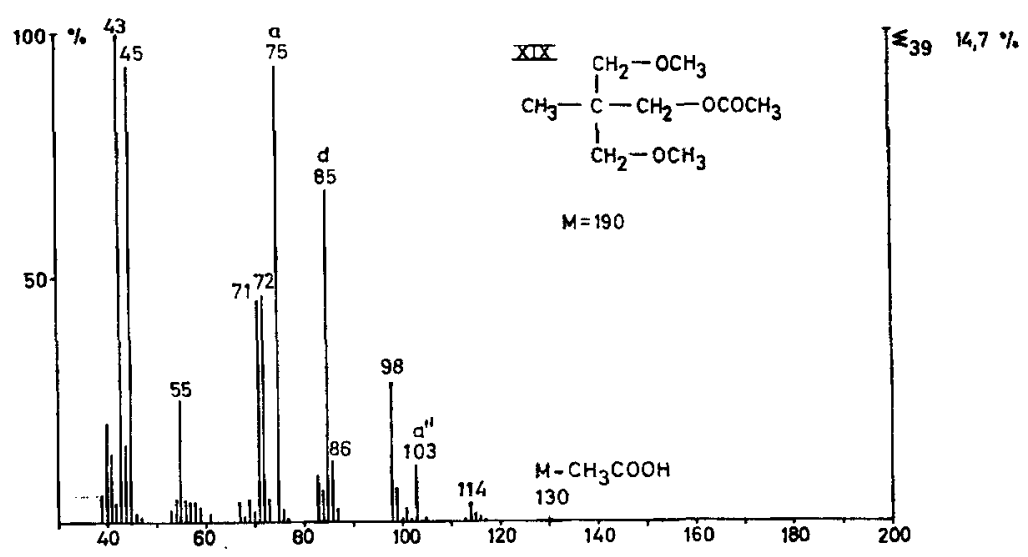

ABr. 12. Massenspektrum des Pentaglycerin-di-O-methyl-monoacetats, $70 \mathrm{eV}$.

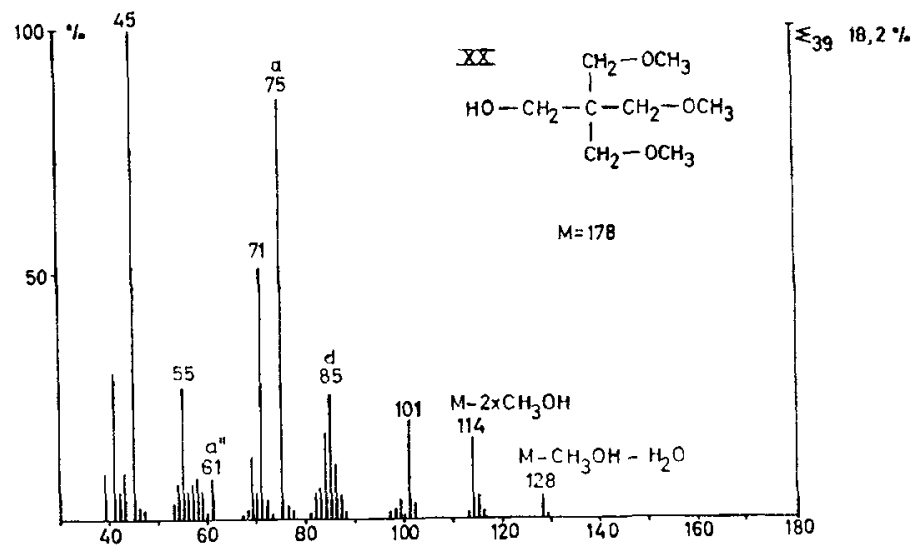

ABB. 13. Massenspektrum des Pentaerythrit-trimethyläthers, $70 \mathrm{eV}$.

Wird aus den Molekül-Ionen von XVIII, XIX und XX nicht $\mathrm{H}_{2} \mathrm{O}$ bzw. $\mathrm{CH}_{3} \mathrm{COOH}$ abgespalten sondern $\mathrm{CH}_{3} \mathrm{OH}$, so entstehen durch analoge Umlagerungsreaktionen Ionen der MZ 61 bzw. der MZ 103. Der Peak der MZ 103 im Massenspektrum von XIX ist klein im Vergleich zu dem der MZ 75, offensichtlich wird aus dem Molekül-Ion viel leichter $\mathrm{CH}_{3} \mathrm{COOH}$ als $\mathrm{CH}_{3} \mathrm{OH}$ abgespalten. Im Gegensatz dazu ist im Massenspektrum von XVIII der Peak der MZ 61 sogar größer als der Peak der MZ 75. Aus dem Molekül-Ion von XVIII wird daher bevorzugt $\mathrm{CH}_{3} \mathrm{OH}$ eliminiert. Das Massenspektrum von XX besitzt einen deutlichen Peak der Ionen $\left[\mathrm{M}-2 \mathrm{xCH}_{3} \mathrm{OH}\right]$, aber 
nur einen kleinen Peak der Ionen [ $\left.\mathrm{M}-\left(\mathrm{CH}_{3} \mathrm{OH}+\mathrm{H}_{2} \mathrm{O}\right)\right]$. Auch aus dem MolekülIon von $\mathrm{XX}$ wird daher bevorzugt $\mathrm{CH}_{3} \mathrm{OH}$ abgespalten. Der Peak der Ionen der $m / e 61$ ist hier jedoch klein. Dies zeigt, daß in dem Radikal-Ion [M- $\left.\mathrm{CH}_{3} \mathrm{OH}\right]$ bevorzugt eine $\mathrm{CH}_{3} \mathrm{O}$-Gruppe wandert und nicht die HO-Gruppe:

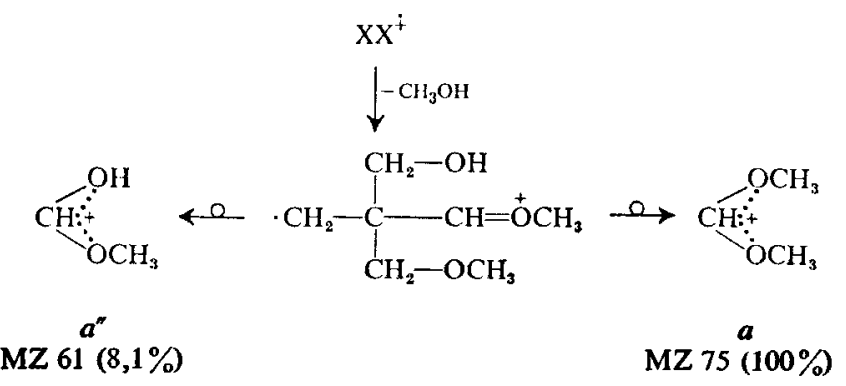

Interessant ist das Massenspektrum des Dimethyläthers von 2-Methyl-2-chlormethylpropan-1,3-diol (XXI, Abb. 14). Der Cl-Substituent einer aliphatischen Verbindung kann in einer 1,3-Eliminierung als $\mathrm{HCl}$ abgespalten werden. ${ }^{11}$ Trotzdem zeigt das Massenspektrum von XXI weder einen größeren Peak der Umlagerungsionen $a$ noch einen der analogen chlorhaltigen Umlagerungsionen der $M Z$ 79/81. Dafür wird wie bei (XV) ein deutlicher Peak für die Ionen $\left[\mathrm{M}-\mathrm{CH}_{3} \mathrm{OH}\right]$ bei den $\mathrm{MZ}$ 134/136

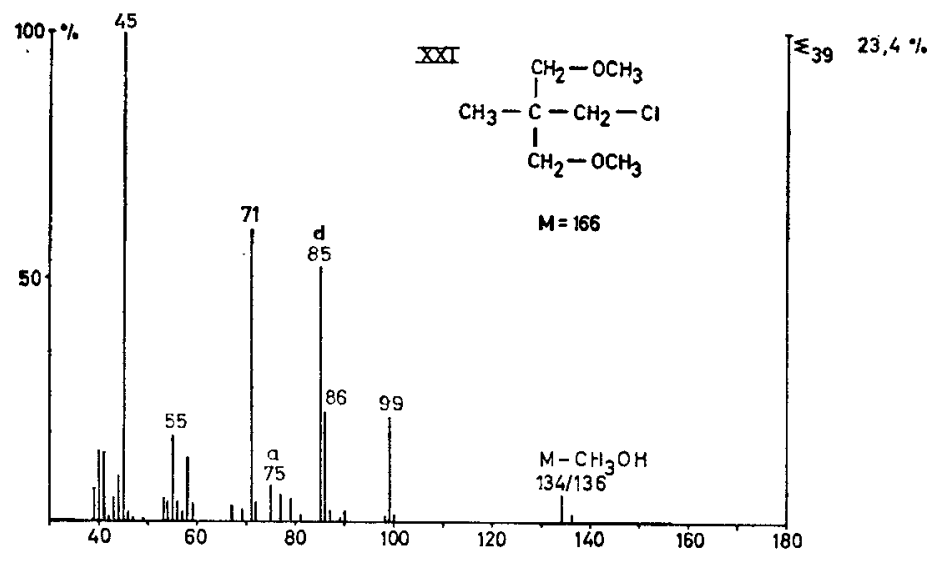

ABB. 14. Massenspektrum des 2-Chlormethyl-2-methyl-propan-1.3-diol-dimethyläthers, $70 \mathrm{eV}$.

beobachtet, aber kein Peak für die Tonen $[\mathrm{M}-\mathrm{HCl}]$. Die Abspaltung von $\mathrm{HCl}$ aus den Molekül-Ionen erfolgt offensichtlich viel langsamer als die Eliminierung von $\mathrm{CH}_{3} \mathrm{OH}$. Die Dissoziationsenergie einer $\mathrm{C}-\mathrm{H}$-Bindung an einem mit einer Methoxygruppe substituierten C-Atom ist geringer als an einem $\mathrm{Cl}$-substituierten $\mathrm{C}$-Atom, ${ }^{9}$ so daß in dem Radikal-Ion $\left[\mathrm{M}-\mathrm{CH}_{3} \mathrm{OH}\right]$ die positive Ladung an dem $\mathrm{CH}_{3} \mathrm{O}$ substituierten C-Atom lokalisiert ist. In diesem Radikal-Ion tritt keine Wanderung 
des Cl-Atoms ein:

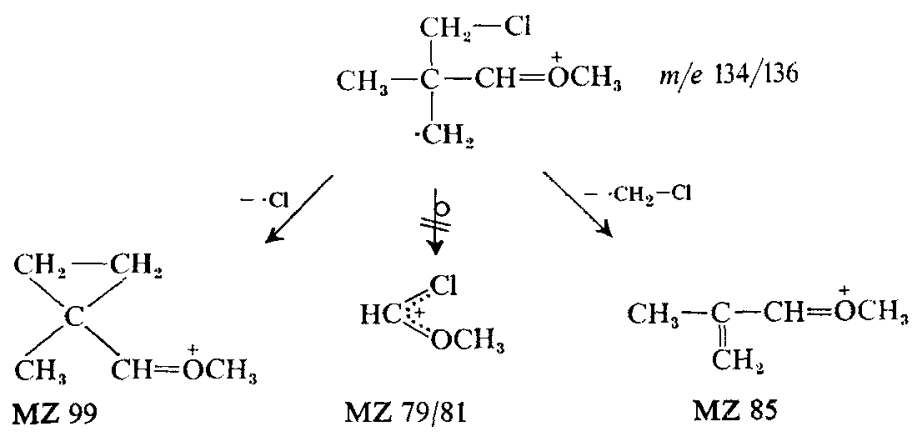

Die Wanderung von Halogenatomen ist beim Zerfall von geradelektronischen Fragment-Ionen beobachtet worden. ${ }^{4}$ In diesen Systemen wird durch die Umlagerung die Eliminierung kleiner neutraler Moleküle ermöglicht. Das aus XXI gebildete Radikal-Ion $\left[\mathrm{M}-\mathrm{CH}_{3} \mathrm{OH}\right]$ zerfällt dagegen durch Abspaltung eines $\mathrm{Cl}$-Atoms und eines $\mathrm{ClCH}_{2}$-Radikals zu Ionen der MZ 99 bzw. MZ 85. Das Massenspektrum von XXI besitzt einen großen Peak bei der MZ 71. Ein metastabiler Peak (gef. 58,6, berechn. 58,62) zeigt, daß diese Ionen durch Abspaltung eines Methylradikals aus Ionen der MZ 86 entstehen:<smiles>C[O+]=CC=CC#CC1CC(C)(C)C1</smiles>

MZ 86

MZ 71

Größere Peaks der MZ 71 und metastabile Peaks bei der MZ 58,6 findet man auch in den Massenspektren von XVI bis XX. Bei XVIII entsteht der Peak der MZ 71 z.T. durch Ionen des Typs MZ 85 mit $\mathrm{OH}$ statt $\mathrm{OCH}_{3}$. Diese Verbindung besitzt jedoch als einzige auch einen größeren Peak der MZ 57. Die Ionen der MZ 86, die die Vorstufe der Ionen der MZ 71 sind, entstehen formal durch die Abspaltung eines Y-Substituenten und eines $\mathrm{CH}_{3} \mathrm{OCH}_{2}$-Radikals (bzw. $\mathrm{CH}_{3} \mathrm{O}$ - und $\mathrm{YCH}_{2}$-Radikals). Will man die Formulierung der energetisch ungünstigen doppelten Radikalabspaltung aus den Molekül-Ionen vermeiden, so muß die Eliminierung von $\mathrm{YCH}_{2} \mathrm{OCH}_{3}$ angenommen werden:<smiles>[Y]C(OC)[C@H]([Y])OC</smiles>

MZ 86

Ungeklärt ist die Beobachtung, daß im Massenspektrum von XIX zwar Ionen der

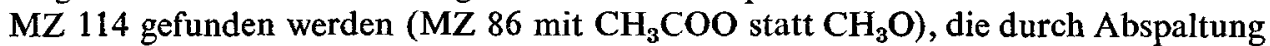
von Keten zu Ionen der MZ 72 zerfallen, daß aber im Gegensatz zu XVIII keine weitere Reaktion unter Abspaltung eines Methylradikals zu Ionen der MZ 57 eintritt. 


\section{EXPERIMENTELLER TEIL}

Methyläther. Die Polyole wurden mit $\mathrm{NaH}$ in Tetrahydrofuran in die Na-Alkoholate überführt, das Tetrahydrofuran im Vakuum abdestilliert, und der Rückstand mit überschüssi sem Methyljodid 24 Stunden am Rückflußkuihler erhitzt. ${ }^{12}$ Die Methyläther wurden durch fraktionierte Destillation isoliert und gaschromatographisch analysiert. Die einzelnen Fraktionen enthie]ten einen unterschiedlichen Gehalt an partiell methylierten Derivaten. Eine vollständige Abtren uung der partiell methylierten Verbindungen durch Destillation gelang in den meisten Fällen nicht.

Trideuteromethyläther der Polyole wurden analog durch Umsetzen mit Tride ıteromethyljodid dargestellt.

Glycerin-1,3-di-O-trideuteromethyl-2-O-methyläther (IXb) wurde aus Glycerin-2-O-methyläther (dargestellt aus 1,3-Benzyliden-glycerin ${ }^{13}$ ) durch Trideuteromethylierung erhalten.

Pentan-1,2,3-triol-trimethyläther- $d_{1}-(3) .3 \mathrm{gr}$. Äthyl-vinyl-keton wurden in der Wärme in $100 \mathrm{ml}$ $\mathrm{H}_{2} \mathrm{O}$ gelöst und mit $20 \mathrm{ml}$ Perhydrol unter Zusatz von $0,1 \mathrm{gr}$. Wolframtrioxid bei 7$)^{\circ} \mathrm{C}$ hydroxyliert. Der Rückstand (1,6 gr) wurde nach Abfiltrieren des Katalysators und Eindampfen :m Vakuum ohne weitere Reinigung in Tetrahydrofuran gelöst und mit LiAID 4 reduziert. Ubersihussiges LiAID $_{4}$ wurde durch feuchtes Tetrahydrofuran zerstört, das Tetrahydrofuran abgedampf stand mehrmals mit warmen Essigester extrahiert. AnschlieBende Destillation erga, 1,1 gr. Pentan1,2,3-triol-d $\mathrm{d}_{1}-(3)$, das wie üblich methyliert wurde.

Gaschromatographische Analyse und Trennung der permethylierten Polyole zu r Aufnahme der Massenspektren wurde auf einem F6-Gaschromatographen (Perkin-Elmer, Co) mit einer $50 \mathrm{~m}$ R-Kapillarsäule durchgeführt. (Trägergasstrom 1,2 ml He, Teilungsverhältnis 1:100, Säulentemperatur $100^{\circ}, 130^{\circ}, 150^{\circ}$ und $175^{\circ}$ ). Unter diesen Bedingungen werden permethylierte und partiell methylierte Derivate der Polyole einwandfrei getrennt, außerdem auch die Disstereomeren der Trimethyläther von X, XI, XIII und XIV. Eine vollständige Trennung der versch edenen isomeren Dimethyläther der Triole gelang jedoch nicht.

Massenspektren wurden mit einem CH4-Massenspektrometer (Varian Mat, Brimen) aufgenommen. Der Einlaß der Substanzen erfolgte durch eine Kopplung des Massenspektrometers mit der Kapillarsäule des Gaschromatographen über ein auf $150^{\circ}$ beheiztes CS2-Kapillarsys em (Varian Mat, Bremen).

Massefeinbestimmungen wurden mit einem SM-1-Massenspektrometer (Variar MAT, Bremen) bei einem Auflösungsvermögen von 12000 (10\% Tal) nach der 'peak matchin ;' Methode ausgeführt. Der Probeneinlaß erfolgte über ein auf $150^{\circ}$ geheiztes HTE-System.

Herrn Prof. Dr. K. Heyns danken wir für seinen Rat und seine Unterstützun!; bei der Durchführung dieser Untersuchungen. Dem Fond der chemischen Industrie sind wir ür Sachbeihilfen zu Dank verpflichtet.

\section{LITERATUR}

1. K. Heyns, D. Müller, Tetrahedron 21, 55 (1965).

2. H. F. Grützmacher, J. Winkler, K. Heyns Tetrahedron Letters 6051 (1966).

3. a: M. M. Green, D. S. Weinberg und C. Djerassi, J. Am. Chem. Soc. 88, 388: (1966). b: M. M. Green und C. Djerassi, J. Am. Chem. Soc. 89, 5190 (1967).

4. R. G. Cooks, J. Ronayne und D. H. Williams, J. Chem. Soc. 2601 (1967).

5. D. C. DeJongh, c 27 in 153rd A.C.S. Meeting Abstr., April 1967.

6. K. Heyns und D. Müller, Tetrahedron Letters 6061 (1966).

7. M. Vandewalle, N. Schamp und M. Francque, Bull. Soc. Chim. Belges 75, $84\{$ (1966).

8. K. Heyns, H. F. Grützmacher, H. Scharmann und D. Müller, Fortschr. Chein. Forsch. 5, 448 (1966).

9. R. H. Martin, F. W. Lampe und R. W. Taft J. Am. Chem. Soc. 88, 1353 (196i).

10. Unveröffentlichte Ergebnisse von H. Scharmann aus dem Chemischen staatsinstitut der Universität Hamburg.

11. A. M. Duffield, S. D. Sample und C. Djerassi, Chem. Commun. 193 (1966)

12. D. M. Lemal, P. D. Pacht und R. B. Woodward, Tetrahedron 18, 1275 (1962)

13. H. S. Hill, M. S. Whelen und H. Hibbert, J. Am. Chem. Soc. 50, 2235 (1928). 